\title{
BUBBLE FORMATION IN HOT NUCLEI INDUCED BY STATISTICAL FLUCTUATIONS
}

\author{
A.H. BLIN, M. BRACK and B. HILLER \\ Institut für theoretische Physik, Universität Regensburg, D-8400 Regensburg, Fed. Rep. Germany \\ Received 10 July 1986
}

\begin{abstract}
A semiclassical study of bubble formation (nucleation) in nuclei at finite temperature is presented. The effects of the Coulomb interaction and of the density profile parametrization are investigated. Significant nucleation probabilities are found.
\end{abstract}

Since the equation of state of hot nuclear matter is of van der Waals type, one might expect that hot nuclei exhibit phase transitions. Their existence has been conjectured in experiments with high-energy protons on nuclei and in heavy-ion collisions $[1,2]$. The role of statistical and quantum fluctuations which lead to phase transitions has been first analyzed in a microscopic approach in ref. [3], based on relativistic mean field theory of nuclei [4]. In a recent work [5], a similar approach, also using the path integral technique to calculate phase transitions, has been applied to bubble formation in hot and dense nuclear matter. In this work we improve upon ref. [5] by including Coulomb forces which are of great importance in realistic calculations. In addition, we use a more appropriate parametrization of the bubble density profiles and take gradient corrections to the free kinetic energy functional into account.

The picture we have in mind is that hot compressed nuclear matter produced in a heavy-ion reaction, may by subsequent (isentropic) expansion reach the region of metastability, where bubbles of lower than liquid density may be formed. The formation of droplets in a gas background can be excluded by the argument that the gas density is below freeze out. The phase transitions are due to statistical fluctuations. Quantum fluctuations have been shown to be

* Work supported by Deutsche Forschungsgemeinschaft, grant no. $\mathrm{Br} 733 / 4-1$. relevant only for temperatures $T \lesssim 1 \mathrm{MeV}[5]^{\ddagger 1}$. The transition probability $W$ from a metastable phase to a stable one has a WKB-like form

$W \propto \exp \left(-\widetilde{\Omega}_{\mathrm{c}}^{\max } / T\right)$,

where $\widetilde{\Omega}_{\mathrm{c}}^{\max }$ is the maximum of the thermodynamical potential [3,5], see eq. (3) and fig. 1 . We normalize $W$ to 1 at vanshing potential barrier [5].

The relevant potential for isothermal processes in a grand canonical ensemble is the thermodynamical potential $\Omega$, defined as

$\Omega=E-T S-\mu N$,

where $F=E-T S$ is the free energy calculated from the internal energy $E$ and the entropy $S$ at given temperature $T$. The chemical potential $\mu$ is the Lagrange multiplier of the particle number $N$. We assume a homogeneous liquid background density $\rho_{\mathrm{L}}$, on which bubble formation can take place. Therefore we introduce a thermodynamical potential $\Omega$, in which the constant potential associated with the homogeneous background $\Omega\left[\rho_{\mathrm{L}}\right]$ is subtracted.

The thermodynamical treatment of charged systems, however, is a difficult issue, since the Coulomb interaction is a long-range force and hence has no clear place in thermodynamics. We adopt the subtraction prescription of refs. [6-8] where the thermodynamical potential for a charged bubble takes the

\# This holds true also for the present calculation, as the mass parameters resulting from eq. (5) are almost the same as in ref. [5]. 
form

$\widetilde{\Omega}_{\mathrm{c}}=\Omega[\rho]-\Omega\left[\rho_{\mathrm{L}}\right]-E_{\mathrm{c}}\left[\rho-\rho_{\mathrm{L}}\right]$,

$\rho$ is the density profile of the bubble and

$E_{\mathrm{c}}=\frac{e^{2}}{8} \int \mathrm{d}^{3} r_{1} \mathrm{~d}^{3} r_{2} \frac{\left[\rho\left(r_{1}\right)-\rho_{\mathrm{L}}\right]\left[\rho\left(r_{2}\right)-\rho_{\mathrm{L}}\right]}{\left|\boldsymbol{r}_{1}-\boldsymbol{r}_{2}\right|}$.

Eq. (4) describes the Coulomb energy content of the bubble alone, i.e. excludes the contribution of the background and of the relative interaction between background and bubble. Exchange terms of the Coulomb energy are neglected and equal proton and neutron densities are assumed (so that $\rho_{\mathrm{p}}=\rho / 2$ ).

The density of a bubble is parametrized in spherical symmetry in analogy to ref. [9] as

$\rho\left(r_{\mathrm{l}} R\right)=\rho_{\mathrm{L}}+\frac{\left[\rho_{\mathrm{G}}(R)-\rho_{\mathrm{L}}\right][1+\cosh (R / a)]}{\cosh (R / a)+\cosh (r / a)}$,

where $R$ is the radius parameter of the bubble and $a$ its surface thickness. The gas density $\rho_{\mathrm{G}}(R)$ is extracted from ref. [9] and describes the dependence of the central bubble density on the radius. As the radius parameter $R$ goes to zero or plus infinity, the density $\rho(r, R)$ approaches the homogeneous liquid $\rho_{\mathrm{L}}$ and the gas $\rho_{\mathrm{G}}(\infty)$ density, respectively. The values $\rho_{\mathrm{L}}$ and $\rho_{\mathrm{G}}(\infty)$ are taken as in ref. [5] and describe the homogeneous liquid and gas phases at equal chemical potential $\mu$ and temperature $T$. In our present case of interest, $\rho_{\mathrm{L}}$ is metastable in the sense that the corresponding thermodynamical energy density has a higher minimum at $\rho_{\mathrm{L}}$ than at $\rho_{\mathrm{G}}(\infty)$. The effective region of metastability is, however, modified in the presence of surface and Coulomb energies of a finite-size bubble, as compared to homogeneous nuclear matter.

We are aware of the fact that the background density is distorted due to the Coulomb interaction, as was found in Hartree-Fock calculations [7]. But since the background contribution is subtracted off, we are confident that our simple parametrization is reasonable. In fact, the subtracted densities of refs. $[7,8]$ do look very much like a parametrization of the form of eq. (5).

The thermodynamical potential eq. (3) is calculated semiclassically for symmetric nuclear matter using the $\mathrm{SkM}^{*}$ force in a finite-temperature extended Thomas-Fermi approximation [10]. We omit the

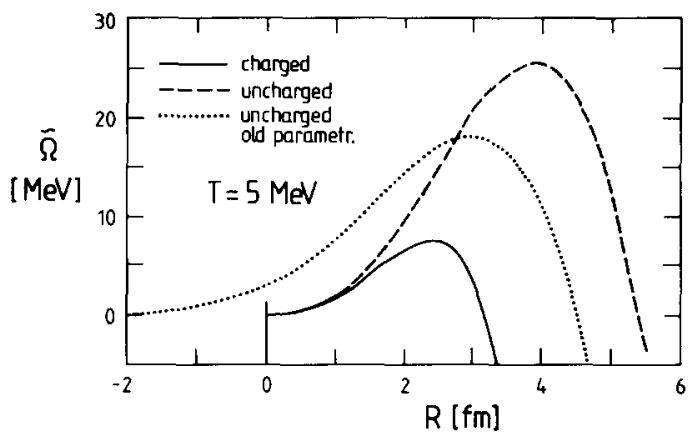

Fig. 1. The thermodynamical potential $\Omega$ as a function of the bubble radius parameter $R$ at $T=5 \mathrm{MeV}$ and $\mu=-20.2 \mathrm{MeV}$, for charged (full line) and uncharged (dashed line) bubbles. The dotted line represents uncharged bubbles with the parametrization of ref. [5].

gradients of the effective mass as well as the spin-orbit terms like in ref. [5], but include the second-order gradient corrections to the free kinetic energy functional at finite temperature (Weizsäckerlike term).

In fig. 1 we compare the thermodynamical potentials $\Omega$ as functions of the bubble radius $R$ calculated at $T=5 \mathrm{MeV}$ and chemical potential $\mu=-20.2 \mathrm{MeV}$ with (solid line) and without (dashed line) Coulomb interaction, taking a surface thickness of $a=1$ $\mathrm{fm}$. The barrier which exists between the liquid $(R=0)$ and gas $(R \rightarrow \infty)$ phases is due to an interplay of volume and surface energies (and Coulomb energy, if present). The Coulomb energy influences the results by diminishing the barrier and shifting the critical radius $R_{\mathrm{cr}}$ (at which $\widetilde{\Omega}$ has a maximum) to smaller values. This happens because the Coulomb energy favors the gas phase, since a bubble has a "missing charge" as compared to the liquid phase. The dotted line in fig. 1 corresponds to the uncharged bubbles in the parametrization of ref. [5], where the radius parameter $R$ is defined down to $-\infty$. This parametrization has the unrealistic feature that for small $|R|$ the density profile exhibits a cusp at $r=0$, and thus distorts the surface energy.

Fig. 2 presents the statistical phase transition probability $W$, eq. (1), as a function of the background density $\rho_{\mathrm{L}}$ in eq. (5) which is uniquely related to the chemical potential $\mu$. Phase transitions are substantially enhanced for charged bubbles (solid line) as compared to the uncharged case (dashed line). For $\rho_{\mathrm{L}} \rightarrow 0.95 \mathrm{fm}^{-3}$, the barriers of $\widetilde{\Omega}$ vanish, 


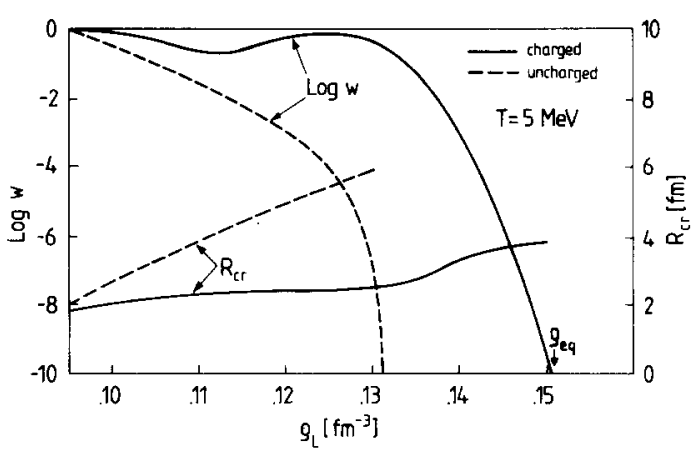

Fig.2. The bubble formation probability $W$ at $T=5 \mathrm{MeV}$ (left scale) as a function of the liquid background density $\rho_{\mathrm{L}}$ for charged (solid line) and uncharged (dashed line) systems. Right scale: the corresponding critical radii $R_{\text {cr }}$.

since at this value of $\rho_{\mathrm{L}}$ the homogeneous phase at $\rho=\rho_{\mathrm{L}}$ has no energy minimum any more. As $\rho_{\mathrm{L}}$ approaches $\rho_{\text {eq }}$, the potentials of the homogeneous phases $\rho=\rho_{\mathrm{L}}$ and $\rho=\rho_{\mathrm{G}}(\infty)$ become the same. Therefore the volume energy contribution of $\widehat{\Omega}$ vanishes and due to the positive surface energy $\widetilde{\Omega}$ increases monotonically with increasing $R$. Therefore, $W$ drops to zero. The Coulomb energy "postpones" this drop of $W$.

The corresponding critical radii $R_{\mathrm{cr}}$ are also shown (scale on the right); they tend to a finite value $R \approx 2$ $\mathrm{fm}$ as $W \rightarrow 1$ (vanishing barrier of $\widetilde{\Omega}$ ). The dips in both the $W$ and $R_{\text {cr }}$ curves are due to an interplay of the various contributions to the energy functional.

The qualitative behavior of $W$ remains similar as that for $T=5 \mathrm{MeV}$ for smaller or higher temperatures. Quantitatively, $W$ drops less rapidly as a function of $\rho_{\mathrm{L}}$ as the temperature increases. In conclusion we note, that if in a heavy-ion collision a hot nucleus reaches a metastable density below a certain value, our model predicts a probability of order 1 to form bubbles due to statistical fluctuations. The Coulomb energy is seen to enhance the transition probability by orders of magnitude. The real scenario will depend of course on the dynamics of the reaction.

\section{References}

[1] M.W. Curtin, H. Toki and D.K. Scott, Phys. Lett. B 123 (1983) 289.

[2] A.D Panagiotou et al., Phys. Rev. Lett. 52 (19840 496.

[3] H. Reinhardt and H. Schulz, Nucl. Phys. A 432 (1985) 630.

[4] J.D. Walecka, Ann. Phys. 83 (1974) 491; Phys. Lett. B 59 (1975) 109; S.A. Chin, Ann. Phys. 108 (1977) 301.

[5] A.H. Blin, B. Hiller, H. Reinhardt and P. Schuck, submitted to Nucl. Phys.

[6] P. Bonche, S. Levit and D. Vautherin, Nucl. Phys. A 427 (1984) 278.

[7] P. Bonche, S. Levit and D. Vautherin, Nucl. Phys. A 436 (1985) 265 .

[8] E. Suraud, preprint ISN Grenoble (February 1986).

[9] P. Gleißl et al., to be published.

[10] M. Brack, C. Guet and H.-B. Håkansson, Phys. Rep. 123 (1985) 275. 